\title{
THREE-DIMENSIONAL WAVE POLYNOMIALS
}

\author{
ARTUR MACIA̧G \\ Received 16 April 2004 and in revised form 14 September 2004
}

We demonstrate a specific power series expansion technique to solve the three-dimensional homogeneous and inhomogeneous wave equations. As solving functions, so-called wave polynomials are used. The presented method is useful for a finite body of certain shape. Recurrent formulas to improve efficiency are obtained for the wave polynomials and their derivatives in a Cartesian, spherical, and cylindrical coordinate system. Formulas for a particular solution of the inhomogeneous wave equation are derived. The accuracy of the method is discussed and some typical examples are shown.

\section{Introduction and notation}

A linear wave equation can be solved using different methods. Some of them are better for infinite bodies and others for finite bodies but of simple shape. The method presented here is useful for finite bodies but the shape of the body can be more complicated. The key idea of the method is to find functions (polynomials) satisfying a given differential equation to be fitted to the governing initial and boundary conditions. In this sense it is a variant of the Trefftz method $[13,15]$. Especially, in spherical and cylindrical coordinate system, this method avoids Bessel functions for solution.

The method originates from [12] but only for the case of one-dimensional heatconduction problems in the Cartesian coordinate system. In the same case, the heat polynomials were applied for solving unsteady heat conduction problems in [14]. The method is continued in the Cartesian coordinate system by the contributions [8,9], describing heat polynomials for the two- and three-dimensional case. Application of the heat polynomials in polar and cylindrical coordinates is shown in $[5,6,7]$. A slightly different approach for one-dimensional heat polynomials is presented in [10].

The applications of this method for inverse heat conduction problems are described in $[3,4,5,6,7,8,9]$. The paper [1] contains a highly interesting idea using heat polynomials as a new type of finite element base functions.

All papers described above refer to the heat conduction equation. The work [2] deals with a lot of other cases involving other differential equations, such as the Laplace, Poisson, and Helmholtz equations. Also the one-dimensional wave equation is solved there. 
The solution for two-dimensional wave equation by using wave polynomials is shown in [11].

Important for the application of the method are the properties of Taylor series

$$
f(x+\mathrm{d} x, y+\mathrm{d} y, z+\mathrm{d} z, t+\mathrm{d} t)=f(x, y, z, t)+\frac{\mathrm{d} f}{1 !}+\frac{\mathrm{d}^{2} f}{2 !}+\cdots+\frac{\mathrm{d}^{N} f}{N !}+R_{N+1},
$$

where

$$
\mathrm{d}^{n} f=\left(\frac{\partial f}{\partial x} \mathrm{~d} x+\frac{\partial f}{\partial y} \mathrm{~d} y+\frac{\partial f}{\partial z} \mathrm{~d} z+\frac{\partial f}{\partial t} \mathrm{~d} t\right)^{n}
$$

Based on this, in Section 2 three-dimensional wave polynomials and their properties in the Cartesian coordinate system are considered. Section 3 contains three-dimensional wave polynomials in the spherical and cylindrical coordinate system. In Section 4, the solution of the wave equation using wave polynomials is obtained. Section 5 discusses the accuracy of the method. Section 6 contains solution for inhomogeneous wave equation and in Section 7 some examples are considered.

\section{Wave polynomials in the Cartesian coordinate system}

They are two ways to obtain wave polynomials. The first one is to use a "generating function." The second is to develop the function satisfying wave equation in Taylor series.

2.1. Generating function. We consider the nondimensional wave equation

$$
\frac{\partial^{2} w}{\partial t^{2}}=\frac{\partial^{2} w}{\partial x^{2}}+\frac{\partial^{2} w}{\partial y^{2}}+\frac{\partial^{2} w}{\partial z^{2}}
$$

The function

$$
g=e^{i(a x+b y+c z+d t)}
$$

satisfying (2.1) when $d^{2}=a^{2}+b^{2}+c^{2}$ is called a generating function for wave polynomials. The power series expansion for (2.2) is

$$
e^{i(a x+b y+c z+d t)}=\sum_{n=0}^{\infty} \sum_{k=0}^{n} \sum_{l=0}^{n-k} \sum_{m=0}^{n-k-l} \tilde{R}_{(n-k-l-m) k l m}(x, y, z, t) a^{n-k-l-m} b^{k} c^{l} d^{m}
$$

where $\tilde{R}_{(n-k-l-m) k l m}(x, y, z, t)$ are polynomials of variables $x, y, z, t$.

Substituting $d^{2}=a^{2}+b^{2}+c^{2}$ in (2.3) we obtain

$$
e^{i(a x+b y+c z+d t)}=\sum_{n=0}^{\infty} \sum_{k=0}^{n} \sum_{l=0}^{n-k} \sum_{\substack{m=0 \\ m<2}}^{n-k-l} R_{(n-k-l-m) k l m}(x, y, z, t) a^{n-k-l-m} b^{k} c^{l} d^{m} .
$$


The real and imaginary parts of polynomials $R$ satisfy (2.1) and are called wave polynomials:

$$
\begin{aligned}
& P_{(n-k-l-m) k l m}(x, y, z, t)=\operatorname{Re}\left(R_{(n-k-l-m) k l m}(x, y, z, t)\right), \\
& Q_{(n-k-l-m) k l m}(x, y, z, t)=\operatorname{Im}\left(R_{(n-k-l-m) k l m}(x, y, z, t)\right),
\end{aligned}
$$

for example,

$$
\begin{aligned}
& P_{0000}=1, \\
& Q_{1000}=x, \quad Q_{0100}=y, \quad Q_{0010}=z, \quad Q_{0001}=t, \\
& P_{2000}=-\frac{x^{2}}{2}-\frac{t^{2}}{2}, \quad P_{1100}=-x y, \quad P_{1010}=-x z, \quad P_{1001}=-x t, \quad P_{0200}=-\frac{y^{2}}{2}-\frac{t^{2}}{2}, \\
& P_{0110}=-y z, \quad P_{0101}=-y t, \quad P_{0020}=-\frac{z^{2}}{2}-\frac{t^{2}}{2}, \quad P_{0011}=-z t, \ldots, \\
& Q_{0000}=P_{1000}=P_{0100}=P_{0010}=P_{0001}=Q_{2000}=Q_{1100}=Q_{1010}=Q_{1001}=\cdots=0 .
\end{aligned}
$$

Note that there is no $R_{0002}$ because $m<2$.

2.2. Partial derivatives of wave polynomials. To obtain recurrent formulas for partial derivatives for wave polynomials we differentiate (2.4):

$$
\frac{\partial g}{\partial x}=i a g=\sum_{n=0}^{\infty} \sum_{k=0}^{n} \sum_{l=0}^{n-k} \sum_{\substack{m=0 \\ m<2}}^{n-k-l} \frac{\partial R_{(n-k-l-m) k l m}}{\partial x} a^{n-k-l m} b^{k} c^{l} d^{m} .
$$

Hence

$$
\begin{aligned}
& \sum_{n=0}^{\infty} \sum_{k=0}^{n} \sum_{l=0}^{n-k} \sum_{\substack{m=0 \\
m<2}}^{n-k-l} i R_{(n-k-l-m) k l m} a^{n-k-l-m+1} b^{k} c^{l} d^{m} \\
& \quad=\sum_{n=0}^{\infty} \sum_{k=0}^{n} \sum_{\substack{l=0 \\
n-k}}^{n-k-l} \frac{\partial R_{(n-k-l-m) k l m}}{\partial x} a^{n-k-l-m} b^{k} c^{l} d^{m}, \\
& \frac{\partial R_{(n-k-l-m) k l m}}{\partial x}=i R_{(n-k-l-m-1) k l m},
\end{aligned}
$$

so that finally

$$
\frac{\partial P_{(n-k-l-m) k l m}}{\partial x}=-Q_{(n-k-l-m-1) k l m}, \quad \frac{\partial Q_{(n-k-l-m) k l m}}{\partial x}=P_{(n-k-l-m-1) k l m} .
$$


Similarly, we have

$$
\begin{gathered}
\frac{\partial P_{(n-k-l-m) k l m}}{\partial y}=-Q_{(n-k-l-m)(k-1) l m}, \quad \frac{\partial Q_{(n-k-l-m) k l m}}{\partial y}=P_{(n-k-l-m)(k-1) l m}, \\
\frac{\partial P_{(n-k-l-m) k l m}}{\partial z}=-Q_{(n-k-l-m) k(l-1) m}, \quad \frac{\partial Q_{(n-k-l-m) k l m}}{\partial z}=P_{(n-k-l-m) k(l-1) m}, \\
\frac{\partial P_{(n-k-l) k l 0}}{\partial t}=-Q_{(n-k-l-2) k l 1}-Q_{(n-k-l)(k-2) l 1}-Q_{(n-k-l) k(l-2) 1}, \\
\frac{\partial P_{(n-k-l-1) k l 1}}{\partial t}=-Q_{(n-k-l-1) k 0}, \\
\frac{\partial Q_{(n-k-l) k l 0}}{\partial t}=P_{(n-k-l-2) k l 1}+P_{(n-k-l)(k-2) l 1}+P_{(n-k-l) k(l-2) 1}, \\
\frac{\partial Q_{(n-k-l-1) k l 1}}{\partial t}=P_{(n-k-l-1) k l 0 .} .
\end{gathered}
$$

Starting values for the derivatives (2.9), (2.10), (2.11), and (2.12) are obtained either from (2.6) or directly by putting zero instead of the polynomial in which any of its subscripts takes a negative value.

2.3. Recurrent formulas for wave polynomials. Recurrent formulas are most useful in numerical calculations. The following theorem enables one to get the wave polynomials $P_{(n-k-l-m) k l m}$ and $Q_{(n-k-l-m) k l m}$.

Theorem 2.1. Let $P_{0000}=1$ and let $Q_{0000}=0$. Let $P_{(n-k-l-m) k l m}=Q_{(n-k-l-m) k l m}=0$ when any subscript is negative. Then, the polynomials

$$
\begin{aligned}
P_{(n-k-l) k l 0}=- & \frac{1}{n}\left(x Q_{(n-k-l-1) k l 0}+y Q_{(n-k-l)(k-1) l 0}+z Q_{(n-k-l) k(l-1) 0}\right. \\
+ & \left.t Q_{(n-k-l-2) k l 1}+t Q_{(n-k-l)(k-2) l 1}+t Q_{(n-k-l) k(l-2) 1}\right) \\
P_{(n-k-l-1) k l 1}= & -\frac{1}{n}\left(x Q_{(n-k-l-2) k l 1}+y Q_{(n-k-l-1)(k-1) l 1}\right. \\
& \left.+z Q_{(n-k-l-1) k(l-1) 1}+t Q_{(n-k-l-1) k l 0}\right) \\
Q_{(n-k-l) k l 0}= & \frac{1}{n}\left(x P_{(n-k-l-1) k l 0}+y P_{(n-k-l)(k-1) l 0}+z P_{(n-k-l) k(l-1) 0}\right. \\
+ & \left.t P_{(n-k-l-2) k l 1}+t P_{(n-k-l)(k-2) l 1}+t P_{(n-k-l) k(l-2) 1}\right), \\
Q_{(n-k-l-1) k l 1}= & \frac{1}{n}\left(x P_{(n-k-l-2) k l 1}+y P_{(n-k-l-1)(k-1) l 1}\right. \\
& \left.+z P_{(n-k-l-1) k(l-1) 1}+t P_{(n-k-l-1) k l 0}\right)
\end{aligned}
$$

satisfy the wave (2.1). 
Proof. For relation (2.13), we assume that all polynomials on the right-hand side satisfy (2.1). Substituting (2.13) in (2.1) we get

$$
\begin{array}{r}
\frac{\partial Q_{(n-k-l-2) k l 1}}{\partial t}+\frac{\partial Q_{(n-k-l)(k-2) l 1}}{\partial t}+\frac{\partial Q_{(n-k-l) k(l-2) 1}}{\partial t} \\
=\frac{\partial Q_{(n-k-l-1) k l 0}}{\partial x}+\frac{\partial Q_{(n-k-l)(k-1) l 0}}{\partial y}+\frac{\partial Q_{(n-k-l) k(l-1) 0}}{\partial z},
\end{array}
$$

hence according to (2.9), (2.10), (2.11), and (2.12) we have

$$
\begin{aligned}
P_{(n-k-l-2) k l 0} & +P_{(n-k-l)(k-2) l 0}+P_{(n-k-l) k(l-2) 0} \\
= & P_{(n-k-l-2) k l 0}+P_{(n-k-l)(k-2) l 0}+P_{(n-k-l) k(l-2) 0 .}
\end{aligned}
$$

This proves the theorem. The proof for (2.14), (2.15), and (2.16) is similar.

Similarly as before, starting values for the polynomials (2.13)-(2.16) can be obtained either from (2.6) or directly by putting zero instead of the polynomial in which any of its subscripts takes a negative value.

2.4. Expansion of the function satisfying wave equation in Taylor series. Similarly as for other equations [2], the wave polynomials can be obtained using Taylor series (1.1) for function $w$. Let function $w(x, y, z, t)$ satisfy wave equation (2.1), given boundary and initial conditions. We assume that $w$ is differentiable in the neighborhood of $\left(x_{0}, y_{0}, z_{0}, t_{0}\right)$. Let $\hat{x}=x-x_{0}, \hat{y}=y-y_{0}, \hat{z}=z-z_{0}, \hat{t}=t-t_{0}$. Then, the Taylor series for function $w$ and for $N=2$ is

$$
\begin{aligned}
w(x, y, z, t)= & w\left(x_{0}, y_{0}, z_{0}, t_{0}\right)+\frac{\partial w}{\partial x} \hat{x}+\frac{\partial w}{\partial y} \hat{y}+\frac{\partial w}{\partial z} \hat{z}+\frac{\partial w}{\partial t} \hat{t}+\frac{\partial^{2} w}{\partial x^{2}} \frac{\hat{x}^{2}}{2}+\frac{\partial^{2} w}{\partial y^{2}} \frac{\hat{y}^{2}}{2}+\frac{\partial^{2} w}{\partial z^{2}} \frac{\hat{z}^{2}}{2} \\
& +\frac{\partial^{2} w}{\partial t^{2}} \frac{\hat{t}^{2}}{2}+\frac{\partial^{2} w}{\partial x \partial y} \hat{x} \hat{y}+\frac{\partial^{2} w}{\partial x \partial z} \hat{x} \hat{z}+\frac{\partial^{2} w}{\partial x \partial t} \hat{x} \hat{t}+\frac{\partial^{2} w}{\partial y \partial z} \hat{y} \hat{z}+\frac{\partial^{2} w}{\partial y \partial t} \hat{y} \hat{t}+\frac{\partial^{2} w}{\partial z \partial t} \hat{z} \hat{t}+R_{3} .
\end{aligned}
$$

Eliminating the derivative $\partial^{2} w / \partial t^{2}$ by $(2.1)$ yields

$$
\begin{aligned}
w(x, y, z, t)= & w\left(x_{0}, y_{0}, z_{0}, t_{0}\right)+\frac{\partial w}{\partial x} \hat{x}+\frac{\partial w}{\partial y} \hat{y}+\frac{\partial w}{\partial z} \hat{z}+\frac{\partial w}{\partial t} \hat{t}+\frac{\partial^{2} w}{\partial x^{2}}\left(\frac{\hat{x}^{2}}{2}+\frac{\hat{t}^{2}}{2}\right) \\
& +\frac{\partial^{2} w}{\partial y^{2}}\left(\frac{\hat{y}^{2}}{2}+\frac{\hat{t}^{2}}{2}\right)+\frac{\partial^{2} w}{\partial z^{2}}\left(\frac{\hat{z}^{2}}{2}+\frac{\hat{t}^{2}}{2}\right)+\frac{\partial^{2} w}{\partial x \partial y} \hat{x} \hat{y}+\frac{\partial^{2} w}{\partial x \partial z} \hat{x} \hat{z} \\
& +\frac{\partial^{2} w}{\partial x \partial t} \hat{x} \hat{t}+\frac{\partial^{2} w}{\partial y \partial z} \hat{y} \hat{z}+\frac{\partial^{2} w}{\partial y \partial t} \hat{y} \hat{t}+\frac{\partial^{2} w}{\partial z \partial t} \hat{z} \hat{t}+R_{3} .
\end{aligned}
$$

The coefficients succeeding the derivation terms on the right-hand side represent the nonzero wave polynomials (2.6). Similarly, we get polynomials for $N=3,4, \ldots$. 


\section{Wave polynomials in a spherical and cylindrical coordinate systems}

3.1. Spherical coordinate system. To obtain wave polynomials in a spherical coordinate system

$$
x=r \cos \theta \cos \phi, \quad y=r \cos \theta \sin \phi, \quad z=r \sin \theta
$$

we substitute (3.1) in (2.1) to get

$$
\frac{\partial^{2} w}{\partial t^{2}}=\frac{2}{r} \frac{\partial w}{\partial r}+\frac{\partial^{2} w}{\partial r^{2}}-\frac{1}{r^{2} \operatorname{ctg} \theta} \frac{\partial w}{\partial \theta}+\frac{1}{r^{2}} \frac{\partial^{2} w}{\partial \theta^{2}}+\frac{1}{r^{2} \cos ^{2} \theta} \frac{\partial^{2} w}{\partial \phi^{2}}
$$

Then, to find the wave polynomials in polar coordinates, it is sufficient to substitute (3.1) in polynomials expressed in the Cartesian coordinate system. The polynomials obtained in that way satisfy (3.2), for example, for the nonzero polynomials (2.6),

$$
\begin{array}{llrl}
P_{0000}(r, \phi, \theta, t) & =1, & & Q_{1000}(r, \phi, \theta, t)=r \cos \theta \cos \phi, \\
Q_{0100}(r, \phi, \theta, t) & =r \cos \theta \sin \phi, & & Q_{0010}(r, \phi, \theta, t)=r \sin \theta, \\
Q_{0001}(r, \phi, \theta, t) & =t, & \ldots .
\end{array}
$$

It is obvious that we can use the recurrent formulas (2.9)-(2.12) and (2.13)-(2.16) also in a spherical coordinate system, keeping in mind that $x=r \cos \theta \cos \phi, y=r \cos \theta \sin \phi$, $z=r \sin \theta$.

3.2. Cylindrical coordinate system. To obtain wave polynomials in a cylindrical coordinate system

$$
x=r \cos \phi, \quad y=r \sin \phi, \quad z=z,
$$

we substitute (3.4) in (2.1) to get

$$
\frac{\partial^{2} w}{\partial t^{2}}=\frac{\partial^{2} w}{\partial r^{2}}+\frac{1}{r} \frac{\partial w}{\partial r}+\frac{1}{r^{2}} \frac{\partial^{2} w}{\partial \phi^{2}}+\frac{\partial^{2} w}{\partial z^{2}}
$$

Then, to find the wave polynomials in cylindrical coordinates, it is sufficient to substitute (3.4) in polynomials expressed in the Cartesian coordinate system. The polynomials obtained in that way satisfy (3.5), for example, for the nonzero polynomials (2.6),

$$
\begin{array}{rlrl}
P_{0000}(r, \phi, z, t) & =1, & Q_{1000}(r, \phi, z, t) & =r \cos \phi, \\
Q_{0100}(r, \phi, z, t) & =r \sin \phi, & Q_{0010}(r, \phi, z, t) & =z, \\
Q_{0001}(r, \phi, z, t) & =t, & \ldots
\end{array}
$$

Similarly, it is obvious that we can use the recurrent formulas (2.9)-(2.12) and (2.13)(2.16) in a cylindrical coordinate system, keeping in mind that $x=r \cos \phi, y=r \sin \phi$, $z=z$. In a spherical and cylindrical coordinate system, we avoid Bessel functions in the solution. 


\section{Wave polynomial method}

We denote the nonzero polynomials as

$$
\begin{array}{ll}
V_{1}=P_{0000}, & V_{2}=Q_{1000}, \\
V_{6}=P_{2000}, & V_{3}=Q_{0100}, \quad V_{4}=Q_{0010}, \quad V_{5}=P_{0001},
\end{array}
$$

Obviously, we have one polynomial of order zero, four polynomials of order one, nine polynomials of order two, and so on.

The wave polynomial method discussed in this paper belongs to the class of the Trefftz methods. As an approximation of solution for the wave (2.1), we take a linear combination of wave polynomials

$$
w \approx u=\sum_{n=1}^{N} c_{n} V_{n} .
$$

Because all polynomials $V_{n}$ satisfy (2.1), their linear combination satisfies this equation too. The coefficients $c_{n}$ of linear combination (4.2) are chosen such that the error for fulfilling given boundary and initial conditions corresponding to (2.1) is minimized (see Section 7).

\section{Accuracy of approximation}

The wave polynomial method is an approximation method. It is very important to know how big is the error of approximation. Moreover, this method should be convergent. It is easy to specify the error when in approximation (4.2) all polynomials of order zero to $K$ are taken, for example, for $K=0, N=1$, for $K=1, N=1+4=5$, for $K=2, N=$ $1+4+9=14$, and so on. Then the error of approximation is equal to the remainder term in the Taylor series for function $w$ (see relations (2.19) and (2.20)). This means that the wave polynomial method is convergent if $\lim _{N \rightarrow \infty} R_{N}=0$ in the Taylor series of function $w$.

\section{Solution for an inhomogeneous wave equation}

We consider

$$
L(w)=Q(x, y, z, t)
$$

where $L=\partial^{2} / \partial t^{2}-\partial^{2} / \partial x^{2}-\partial^{2} / \partial y^{2}-\partial^{2} / \partial z^{2}$. As an approximation of the solution we take

$$
w \approx u=\sum_{n=1}^{N} c_{n} V_{n}+w_{p} .
$$

Because all polynomials $V_{n}$ satisfy the wave equation (2.1), a linear combination of them satisfy (2.1). Additionally, $w_{p}$ denotes the particular solution for the inhomogeneous wave equation. Boundary and initial conditions determine the coefficients $c_{n}$. 
6.1. Particular solution. When $Q \in C^{S+1}$, we can use a power series for $Q$ and the solution $w_{p}$ can be calculated as

$$
\begin{aligned}
w_{p} & =L^{-1}(Q) \approx L^{-1}\left(\sum_{s=0}^{S} \sum_{n+k+l+m=s} \frac{\partial^{(n+k+l+m)} Q\left(x_{0}, y_{0}, z_{0}, t_{0}\right)}{\partial x^{n} \partial y^{k} \partial z^{l} \partial t^{m}} \frac{\hat{x}^{n} \hat{y}^{k} \hat{z}^{l} \hat{t}^{m}}{n ! k ! l ! m !}\right) \\
& =\sum_{s=0}^{S} \sum_{n+k+l+m=s} a_{n k l m} L^{-1}\left(\hat{x}^{n} \hat{y}^{k} \hat{z}^{l} \hat{t}^{m}\right),
\end{aligned}
$$

where $\hat{x}=x-x_{0}, \hat{y}=y-y_{0}, \hat{z}=z-z_{0}, \hat{t}=t-t_{0}$.

The coefficients $a_{n k l m}$ are known, when function $Q$ is given. Theorem 6.1 enables to get the particular solution.

Theorem 6.1. Denote $Z_{n k l m}=L^{-1}\left(x^{n} y^{k} z^{l} t^{m}\right)$. Then recurrent formulas for particular solutions are as follows:

$$
\begin{aligned}
Z_{n k l m}^{1}=\frac{1}{(n+2)(n+1)}[ & -x^{n+2} y^{k} z^{l} t^{m}+m(m-1) Z_{(n+2) k l(m-2)} \\
& \left.-k(k-1) Z_{(n+2)(k-2) l m}-l(l-1) Z_{(n+2) k(l-2) m}\right],
\end{aligned}
$$

or

$$
\begin{aligned}
Z_{n k l m}^{2}=\frac{1}{(k+2)(k+1)}[ & -x^{n} y^{k+2} z^{l} t^{m}+m(m-1) Z_{n(k+2) l(m-2)} \\
& \left.-n(n-1) Z_{(n-2)(k+2) l m}-l(l-1) Z_{n(k+2)(l-2) m}\right],
\end{aligned}
$$

or

$$
\begin{aligned}
Z_{n k l m}^{3}=\frac{1}{(l+2)(l+1)}[ & -x^{n} y^{k} z^{l+2} t^{m}+m(m-1) Z_{n k(l+2)(m-2)} \\
& \left.-n(n-1) Z_{(n-2) k(l+2) m}-k(k-1) Z_{n(k-2)(l+2) m}\right],
\end{aligned}
$$

or

$$
\begin{aligned}
Z_{n k l m}^{4}=\frac{1}{(m+2)(m+1)}[ & x^{n} y^{k} z^{l} t^{m+2}+n(n-1) Z_{(n-2) k l(m+2)} \\
& \left.+k(k-1) Z_{n(k-2) l(m+2)}+l(l-1) Z_{n k(l-2)(m+2)}\right] .
\end{aligned}
$$


Proof. For relation (6.4) we assume that $L\left(Z_{n k l m}\right)=x^{n} y^{k} z^{l} t^{m}$ for all $Z$ on the right-hand side of relation (6.4). Then we have

$$
\begin{aligned}
& L\left(Z_{n k l m}\right)=L\left(\frac { 1 } { ( n + 2 ) ( n + 1 ) } \left[-x^{n+2} y^{k} z^{l} t^{m}+m(m-1) Z_{(n+2) k l(m-2)}\right.\right. \\
&\left.\left.-k(k-1) Z_{(n+2)(k-2) l m}-l(l-1) Z_{(n+2) k(l-2) m}\right]\right) \\
&=\frac{1}{(n+2)(n+1)}[-m(m-1) x^{n+2} y^{k} z^{l} t^{m-2}+(n+2)(n+1) x^{n} y^{k} z^{l} t^{m} \\
&+ k(k-1) x^{n+2} y^{k-2} z^{l} t^{m}+l(l-1) x^{n+2} y^{k} z^{l-2} t^{m} \\
&+m(m-1) x^{n+2} y^{k} z^{l} t^{m-2}-k(k-1) x^{n+2} y^{k-2} z^{l} t^{m} \\
&\left.-l(l-1) x^{n+2} y^{k} z^{l-2} t^{m}\right]=x^{n} y^{k} z^{l} t^{m} .
\end{aligned}
$$

This proves the theorem. The proof for (6.5), (6.6), and (6.7) is similar.

In formulas (6.4)-(6.7), a term on the right-hand side is put to be zero if the corresponding subscript takes a negative value.

\section{Examples}

\subsection{Example 1 (cylindrical coordinate system)}

7.1.1. Formulation of the problem. We consider the testing problem described in a cylinder by

(i) equation

$$
\frac{\partial^{2} w}{\partial t^{2}}=\frac{\partial^{2} w}{\partial x^{2}}+\frac{\partial^{2} w}{\partial y^{2}}+\frac{\partial^{2} w}{\partial z^{2}} \quad(x, y, z) \in D, t \geq 0
$$

where $D=\left\{(x, y, z): x^{2}+y^{2} \leq 1,0 \leq z \leq 1\right\}$,

(ii) initial conditions

$$
w(x, y, z, 0)=\sin (x+y+z), \quad \frac{\partial w(x, y, z, 0)}{\partial t}=\sqrt{3} \cos (x+y+z),
$$

(iii) boundary conditions

$$
\begin{gathered}
w(x, y, 0, t)=\sin (x+y+\sqrt{3} t), \quad w(x, y, 1, t)=\sin (x+y+1+\sqrt{3} t), \\
\left.w(x, y, z, t)\right|_{x^{2}+y^{2}=1,0 \leq z \leq 1}=\sin (x+y+z+\sqrt{3} t) .
\end{gathered}
$$

The exact solution for this problem is $w(x, y, z, t)=\sin (x+y+z+\sqrt{3} t)$, but we solve this problem by using wave polynomials in a cylindrical coordinate system. In a cylindrical coordinate system (3.4) we get

(i) equation

$$
\frac{\partial^{2} w}{\partial t^{2}}=\frac{\partial^{2} w}{\partial r^{2}}+\frac{1}{r} \frac{\partial w}{\partial r}+\frac{1}{r^{2}} \frac{\partial^{2} w}{\partial \phi^{2}}+\frac{\partial^{2} w}{\partial z^{2}} \quad(r, \phi, z) \in D, t \geq 0,
$$

where $D=\{(r, \phi, z): 0 \leq r \leq 1,0 \leq \phi \leq 2 \pi, 0 \leq z \leq 1\}$, 
(ii) initial conditions

$$
\begin{gathered}
w(r, \phi, z, 0)=\sin (r \cos \phi+r \sin \phi+z)=d(r, \phi, z), \\
\frac{\partial w(r, \phi, z, 0)}{\partial t}=\sqrt{3} \cos (r \cos \phi+r \sin \phi+z)=h(r, \phi, z),
\end{gathered}
$$

(iii) boundary conditions

$$
\begin{gathered}
w(r, \phi, 0, t)=\sin (r \cos \phi+r \sin \phi+\sqrt{3} t)=p(r, \phi, t), \\
w(r, \phi, 1, t)=\sin (r \cos \phi+r \sin \phi+1+\sqrt{3} t)=q(r, \phi, t), \\
\left.w(r, \phi, z, t)\right|_{r=1,0 \leq z \leq 1}=\sin (\cos \phi+\sin \phi+z+\sqrt{3} t)=s(\phi, z, t) .
\end{gathered}
$$

The exact solution for this problem is

$$
w(r, \phi, z, t)=\sin (r \cos \phi+r \sin \phi+z+\sqrt{3} t) .
$$

7.1.2. Solution by using wave polynomials. The solution $w(r, \phi, z, t)$ is approximated according to (4.2). Here $V_{n}$ are the wave polynomials in a cylindrical coordinate system. We look for an approximate solution in the time interval $(0, \Delta t)$. The coefficients $c_{n}$ have to be chosen appropriately to minimize the functional

$$
\begin{aligned}
I= & \int_{0}^{1} \mathrm{~d} r \int_{0}^{2 \pi} \mathrm{d} \phi \int_{0}^{1}(\underbrace{[u(r, \phi, z, 0)-d(r, \phi, z)]^{2}}_{\text {cond. }(7.5)}+\underbrace{\left[\frac{\partial u(r, \phi, z, 0)}{\partial t}-h(r, \phi, z)\right]^{2}}_{\text {cond. }(7.6)}) \mathrm{d} z \\
& +\int_{0}^{1} \mathrm{~d} r \int_{0}^{2 \pi} \mathrm{d} \phi \int_{0}^{\Delta t} \underbrace{(\underbrace{[u(r, \phi, 0, t)-p(r, \phi, t)]^{2}}_{\text {ond. }(7.8)}}_{\text {cond. }(7.7)}+\underbrace{[u(r, \phi, 1, t)-q(r, \phi, t)]^{2}}_{\text {cond. }(7.9)}) \mathrm{d} t \\
& +\int_{0}^{2 \pi} \mathrm{d} \phi \int_{0}^{1} \mathrm{~d} z \int_{0}^{\Delta t} \underbrace{[u(1, \phi, z, t)-s(\phi, z, t)]^{2}} \mathrm{~d} t .
\end{aligned}
$$

A necessary condition to minimize the functional $I$ is

$$
\frac{\partial I}{\partial c_{1}}=\cdots=\frac{\partial I}{\partial c_{N}}=0 .
$$

From a linear system of equations (7.12), we obtain coefficients $c_{n}$.

In time intervals $(\Delta t, 2 \Delta t),(2 \Delta t, 3 \Delta t), \ldots$, we proceed analogously. Here, the initial condition for time interval $((m-1) \Delta t, m \Delta t)$ is the value of function $u$ at the end of interval $((m-2) \Delta t,(m-1) \Delta t)$. All results below have been obtained for $\Delta t=1$.

Figure 7.1 shows for $r=0.5, z=0.5$ (a) the exact solution, (b) an approximation by polynomials from order 0 to 4 , and (c) the difference between (a) and (b). It is obvious that the presented approximation is very accurate. 


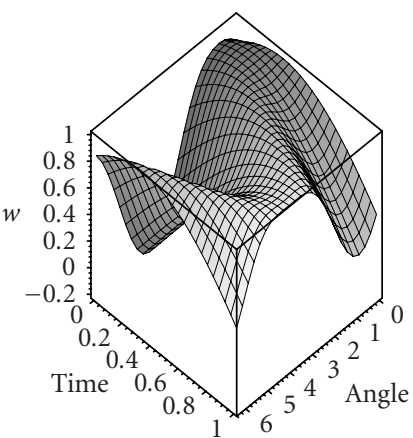

(a)

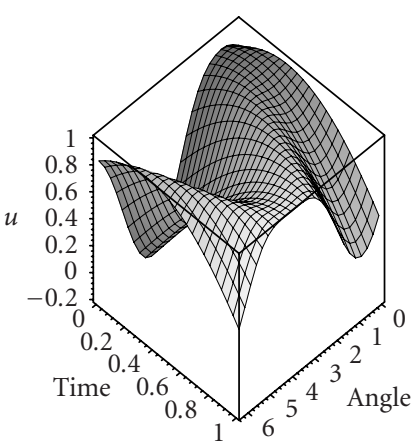

(b)

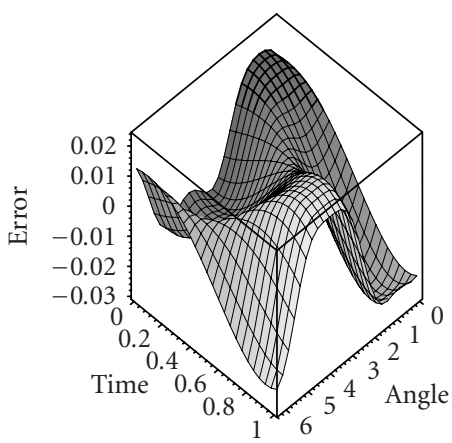

(c)

Figure 7.1. Solution for $r=z=0.5$ : (a) exact, (b) approximation, and (c) difference.

Figure 7.2 shows the exact result as a function of the angle for $x=0.5, y=0.5, t=1$ and the approximation by polynomials from degree 0 to (a) 2, (b) 3, and (c) 4. Again it is obvious that the approximation is very accurate. Figure 7.2 also shows that in a wave polynomial method the error decreases when the degree of polynomials increases.

\subsection{Example 2 (inhomogeneous wave equation)}

7.2.1. Formulation of the problem. We consider the testing problem described in a tetrahedron by

(i) equation

$$
\frac{\partial^{2} w}{\partial t^{2}}=\frac{\partial^{2} w}{\partial x^{2}}+\frac{\partial^{2} w}{\partial y^{2}}+\frac{\partial^{2} w}{\partial z^{2}}+20 \sin (\pi t) \quad(x, y, z) \in D, t \geq 0
$$






(a)

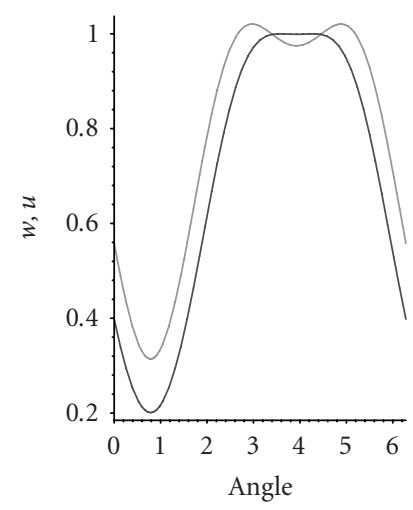

(b)

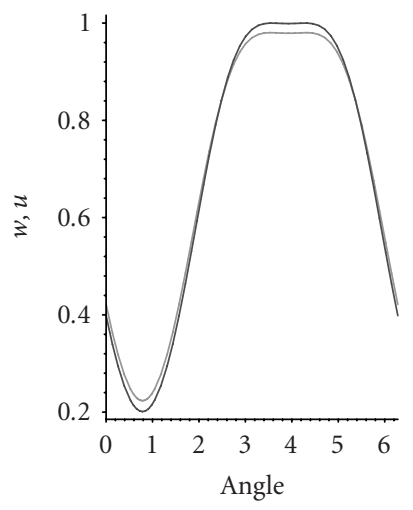

(c)

Figure 7.2. Exact solution versus angle for $x=0.5, y=0.5, t=1$ and approximation by polynomials from degree 0 to (a) 2, (b) 3, and (c) 4 .

where $D=\{(x, y, z): x \geq 0, y \geq 0, z \geq 0, x+y+z \leq 1\}$, here

$$
Q(x, y, z, t)=20 \sin (\pi t)
$$

(ii) initial conditions

$$
\begin{gathered}
w(x, y, z, 0)=\cos (x+y+z)=d(x, y, z), \\
\frac{\partial w(x, y, z, 0)}{\partial t}=-\sqrt{3} \sin (x+y+z)-\frac{20}{\pi}=h(x, y, z),
\end{gathered}
$$


(iii) boundary conditions

$$
\begin{gathered}
w(0, y, z, t)=\cos (y+z+\sqrt{3} t)-\frac{20 \sin (\pi t)}{\pi^{2}}=p(y, z, t), \\
w(x, 0, z, t)=\cos (x+z+\sqrt{3} t)-\frac{20 \sin (\pi t)}{\pi^{2}}=q(x, z, t), \\
w(x, y, 0, t)=\cos (x+y+\sqrt{3} t)-\frac{20 \sin (\pi t)}{\pi^{2}}=r(x, y, t), \\
\left.w(x, y, z, t)\right|_{x+y+z=1}=\cos (1+\sqrt{3} t)-\frac{20 \sin (\pi t)}{\pi^{2}}=s(x, y, t) .
\end{gathered}
$$

The exact solution for this problem is

$$
w(x, y, z, t)=\cos (x+y+z+\sqrt{3} t)-\frac{20 \sin (\pi t)}{\pi^{2}},
$$

but we solve this problem by using wave polynomials in the Cartesian coordinate system. 7.2.2. Solution by using wave polynomials. The solution $w(x, y, z, t)$ is approximated according to (6.2) using (6.3) and (6.4). We look for an approximate solution in the time interval $(0, \Delta t)$. The coefficients $c_{n}$ have to be chosen appropriately to minimize the functional

$$
\begin{aligned}
& I=\int_{0}^{1} \mathrm{~d} x \int_{0}^{1-x} \mathrm{~d} y \int_{0}^{1-x-y} \underbrace{\left[u(x, y, z, 0)+w_{p}(x, y, z, 0)-d(x, y, z)\right]^{2}}_{\text {cond. }(7.15)} \mathrm{d} z \\
& +\int_{0}^{1} \mathrm{~d} x \int_{0}^{1-x} \mathrm{~d} y \int_{0}^{1-x-y} \underbrace{\left[\frac{\partial u(x, y, z, 0)}{\partial t}+\frac{\partial w_{p}(x, y, z, 0)}{\partial t}-h(x, y, z)\right]^{2}}_{\text {cond. }(7.16)} \mathrm{d} z \\
& +\int_{0}^{1} \mathrm{~d} y \int_{0}^{1-y} \mathrm{~d} z \int_{0}^{\Delta t} \underbrace{\left[u(0, y, z, t)+w_{p}(0, y, z, t)-p(y, z, t)\right]^{2}}_{\text {cond. }(7.17)} \mathrm{d} t \\
& +\int_{0}^{1} \mathrm{~d} x \int_{0}^{1-x} \mathrm{~d} z \int_{0}^{\Delta t} \underbrace{\left[u(x, 0, z, t)+w_{p}(x, 0, z, t)-q(x, z, t)\right]^{2}}_{\text {cond. }(7.18)} \mathrm{d} t \\
& +\int_{0}^{1} \mathrm{~d} x \int_{0}^{1-x} \mathrm{~d} y \int_{0}^{\Delta t} \underbrace{\left[u(x, y, 0, t)+w_{p}(x, y, 0, t)-r(x, y, t)\right]^{2}}_{\text {cond. }(7.19)} \mathrm{d} t \\
& +\sqrt{(3)} \int_{0}^{1} \mathrm{~d} x \int_{0}^{1-x} \mathrm{~d} y \int_{0}^{\Delta t} \underbrace{\left[u(x, y, 1-x-y, t)+w_{p}(x, y, 1-x-y, t)-s(x, y, t)\right]^{2}}_{\text {cond.(7.20) }} \mathrm{d} t .
\end{aligned}
$$

A necessary condition to minimize functional $I$ is relation (7.12). Similar to Section 7.1, from linear system of equations (7.12) we obtain coefficients $c_{n}$. All results below are obtained for $\Delta t=1$. 


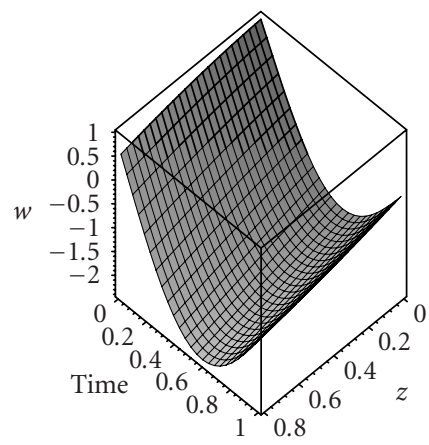

(a)



(b)

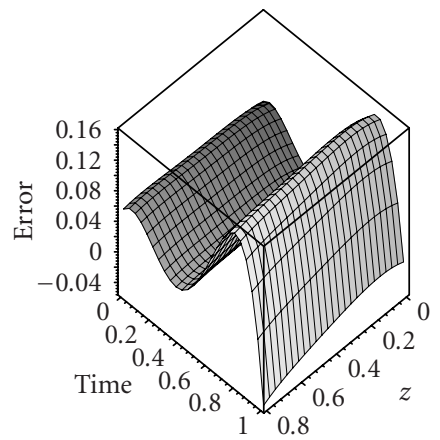

(c)

Figure 7.3. Solution for $x=y=0.1$ : (a) exact, (b) approximation, and (c) difference.

Figure 7.3 shows for $x=0.1, y=0.1$ (a) the exact solution, (b) an approximation by polynomials from order 0 to 4 , and (c) the difference between (a) and (b). It is obvious that the presented approximation is very accurate.

Figure 7.4 shows the exact result for the vibration as a function of time for the location $x=0.1, y=0.1, z=0.1$ and the approximation by polynomials from degree 0 to (a) 1 , (b) 2 , and (c) 4 .

Again, it is obvious that the approximation in time coincides very well. Figure 7.4 shows that in a wave polynomial method for an inhomogeneous wave equation the error decreases when the degree of polynomials increases.

\section{Concluding remarks}

A new technique for solving three-dimensional wave equation has been developed. The main result is to derive formulas for the wave polynomials (satisfying a wave equation) 


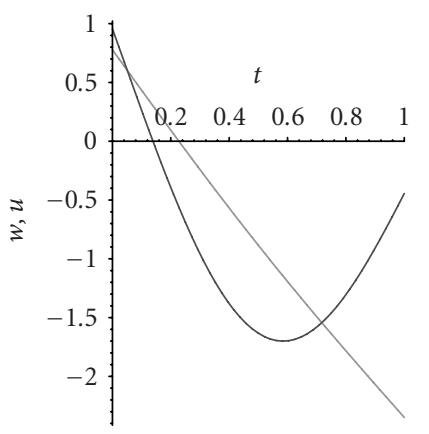

(a)

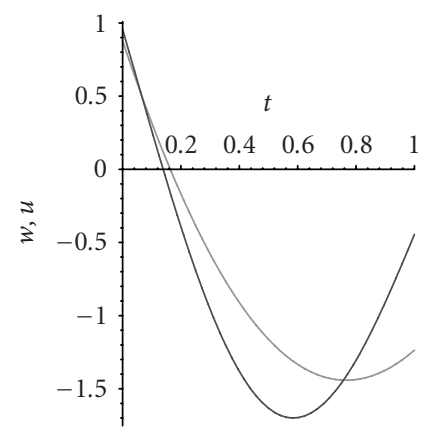

(b)

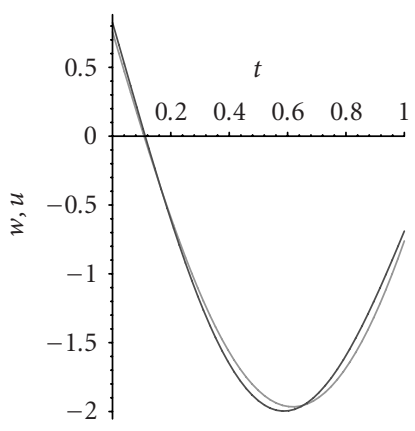

(c)

Figure 7.4. Exact solution versus time for points $x=0.1, y=0.1, z=0.1$ and approximation by polynomials from degree 0 to (a) 1 , (b) 2, and (c) 4 .

and their derivatives. The wave polynomial method presented in this paper is a straightforward method for solving wave equations in finite bodies. This method is also useful when the shape of the body is more complicated. The coefficients $c_{n}$ are determined by calculating integrals-for most shapes it does not create any problem. The method is convergent and the error is equal to the remainder term in the Taylor series. The simple examples presented in the paper show that the obtained approximations of the exact solutions are very good both in the Cartesian and the cylindrical coordinate system. Especially, in the polar and cylindrical coordinate system this method avoids Bessel functions for solution. The solution, as a linear combination of wave polynomials, exactly satisfies the wave equation, approximately initial and boundary conditions. It is important that this method can be used for extrapolation. Therefore, the wave polynomial method can also be applied to inverse problems. Wave polynomials can be used as finite element base functions which will be the subject of another paper. 


\section{Acknowledgments}

The author is an award holder of the NATO Science Fellowships Programme. The author wishes to thank also the University of Karlsruhe, where the paper was written.

\section{References}

[1] M. J. Ciałkowski, Solution of inverse heat conduction problem with use new type of finite element base functions, Proceedings of the International Symposium on Trends in Continuum Physics (B. T. Maruszewski, W. Muschik, and A. Radowicz, eds.), World Scientific Publishing, Singapore, 1999, pp. 64-78.

[2] M. J. Ciałkowski and A. Frạckowiak, Heat Functions and Their Application for Solving Heat Transfer and Mechanical Problems, Poznań University of Technology, Poznań, 2000.

[3] M. J. Ciałkowski, S. Futakiewicz, and L. Hożejowski, Heat polynomials applied to direct and inverse heat conduction problems, Proceedings of the International Symposium on Trends in Continuum Physics (B. T. Maruszewski, W. Muschik, and A. Radowicz, eds.), World Scientific Publishing, Singapore, 1999, pp. 79-88.

[4] - Method of heat polynomials in solving the inverse heat conduction problems, Z. Angew. Math. Mech. 79 (1999), 709-710.

[5] S. Futakiewicz, Heat functions method for solving direct and inverse heat conductions problems, Ph.D. thesis, Poznań University of Technology, Poznań, 1999.

[6] S. Futakiewicz, K. Grysa, and L. Hożejowski, On a problem of boundary temperature identification in a cylindrical layer, Proceedings of the International Symposium on Trends in Continuum Physics (B. T. Maruszewski, W. Muschik, and A. Radowicz, eds.), World Scientific Publishing, Singapore, 1999, pp. 119-125.

[7] S. Futakiewicz and L. Hożejowski, Heat polynomials in solving the direct and inverse heat conduction problems in a cylindrical system of coordinates, Advanced Computational Method in Heat Transfer V, Computational Mechanics Publications, Southampton, 1998, pp. 71-80. , Heat polynomials method in the n-dimensional direct and inverse heat conduction problems, Advanced Computational Method in Heat Transfer V, Computational Mechanics Publications, Southampton, 1998, pp. 103-112.

[9] L. Hożejowski, Heat polynomials and their application for solving direct and inverse heat conductions problems, Ph.D. thesis, University of Technology, Kielce, 1999.

[10] P. Johansen, M. Nielsen, and O. F. Olsen, Branch points in one-dimensional Gaussian scale space, J. Math. Imaging Vision 13 (2000), no. 3, 193-203.

[11] A. Maciagg and J. Wauer, Solution of the two-dimensional wave equation by using wave polynomials, J. Engrg. Math. 51 (2005), no. 4, 339-350.

[12] P. C. Rosenbloom and D. V. Widder, Expansions in terms of heat polynomials and associated functions, Trans. Amer. Math. Soc. 92 (1959), 220-266.

[13] E. Trefftz, Ein Gegenstük zum Ritzschen Verfahren, Proc. 2nd International Congress of Applied Mechanics, Zurich, 1926, pp. 131-137.

[14] H. Yano, S. Fukutani, and A. Kieda, A boundary residual method with heat polynomials for solving unsteady heat conduction problems, J. Franklin Inst. 316 (1983), no. 4, 291-298.

[15] A. P. Zieliński and I. Herrera, Trefftz method: fitting boundary conditions, Internat. J. Numer. Methods Engrg. 24 (1987), no. 5, 871-891.

Artur Maciąg: Department of Mathematics, Faculty of Management and Compuer Modelling, Kielce University of Technology, 25-314 Kielce, Poland

E-mail address: matam@tu.kielce.pl 


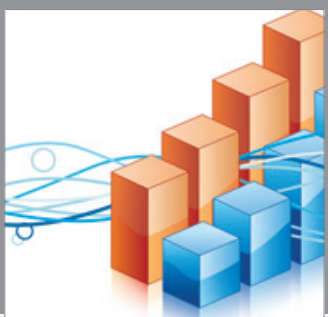

Advances in

Operations Research

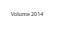

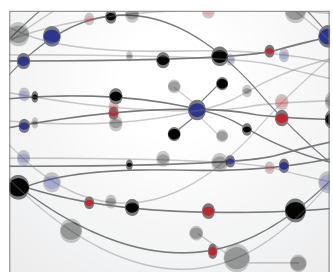

\section{The Scientific} World Journal
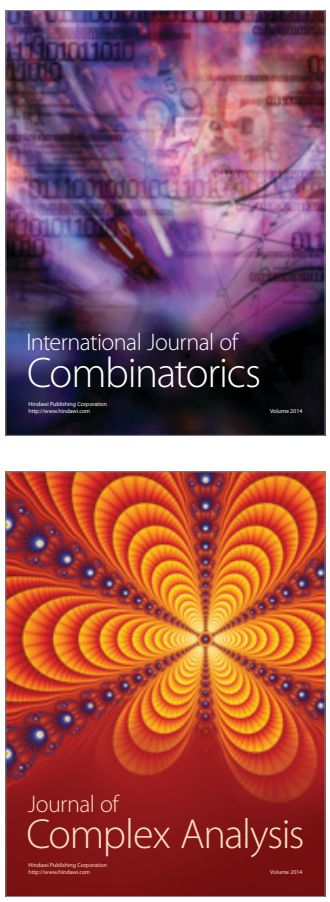

International Journal of

Mathematics and

Mathematical

Sciences
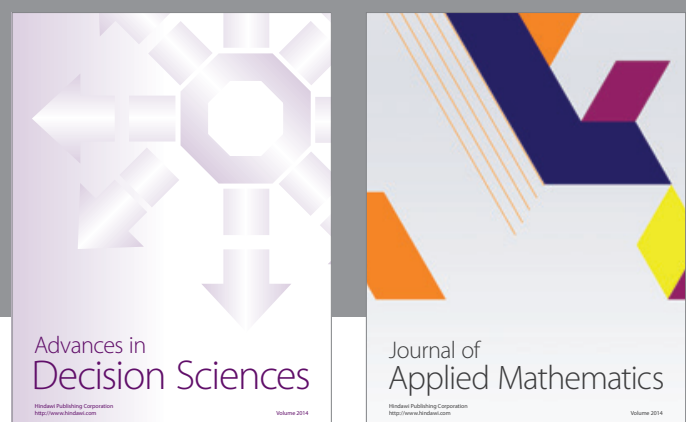

Journal of

Applied Mathematics
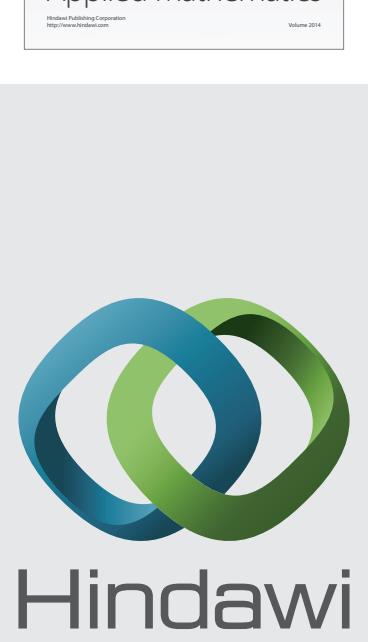

Submit your manuscripts at http://www.hindawi.com
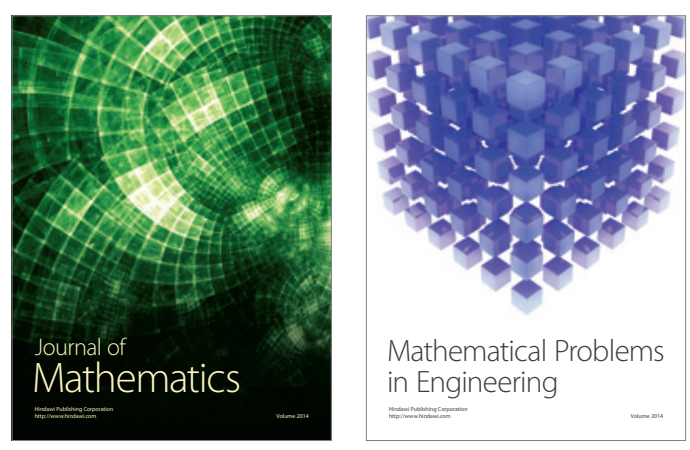

Mathematical Problems in Engineering


Journal of

Function Spaces
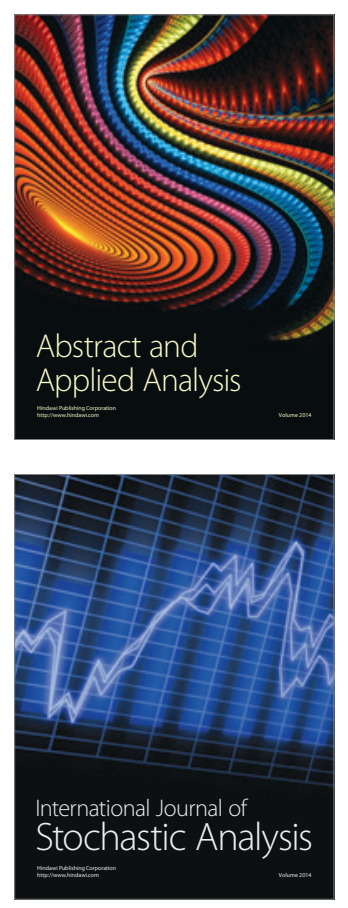

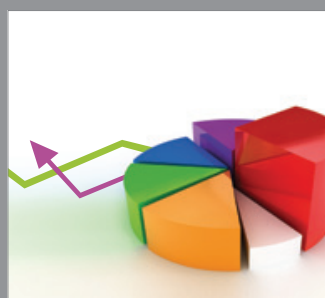

ournal of

Probability and Statistics

Promensencen
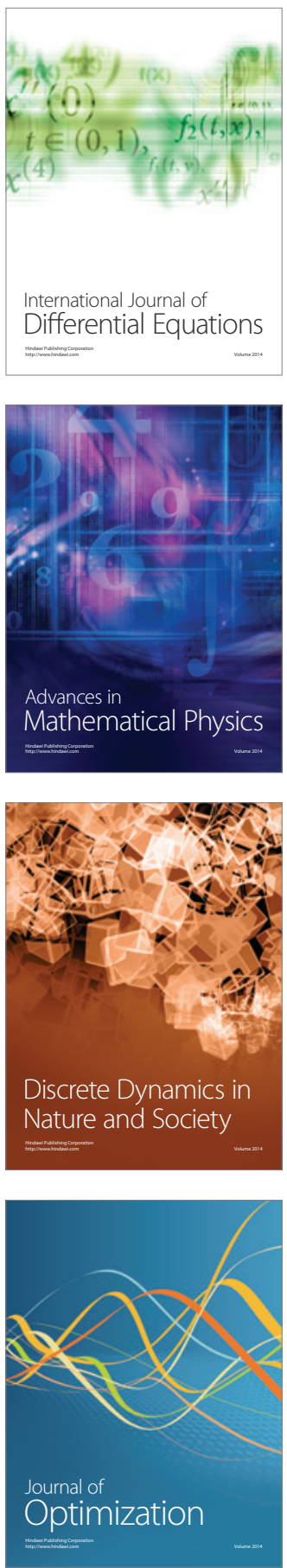